\title{
Pressure-sensitive paint diagnostic to measure species concentration on transpiration-cooled walls
}

\author{
Marc Ewenz Rocher ${ }^{1}$ (1) $\cdot$ Tobias Hermann $^{1} \cdot$ Matthew McGilvray $^{1} \cdot$ Hassan Saad Ifti ${ }^{1}$ Joao Vieira ${ }^{1}$. Chris Hambidge ${ }^{1}$. \\ Mark Kenneth Quinn ${ }^{2} \cdot$ Madeleine Grossman $^{3} \cdot$ Luc Vandeperre $^{3}$
}

Received: 11 October 2021 / Revised: 10 November 2021 / Accepted: 14 November 2021 / Published online: 2 December 2021

(c) The Author(s) 2021

\begin{abstract}
This paper presents the performance of pressure-sensitive paint (PSP) for the direct measurement of species concentration on a porous surface with mass injection. It is used to measure the ability of an injected gas to reduce the mass transfer of freestream species to the surface. A porous alumina sample was sprayed with a PSP luminophore solution. The sample was installed into a flat plate model and exposed to hypersonic cross-flows in the Oxford High-Density Tunnel. Tests were conducted with no coolant injection, air injection, and nitrogen injection at increasing blowing ratios. Oxygen partial pressure maps on the transpiration-cooled surface were obtained for several conditions at unit Reynolds numbers between $2.58-5.0 \times 10^{7} / \mathrm{m}$ and blowing ratios between $0.016-0.078 \%$. The oxygen pressure decreases as the unit Reynolds number decreases and the blowing ratio increases.
\end{abstract}

\section{Graphic abstract}

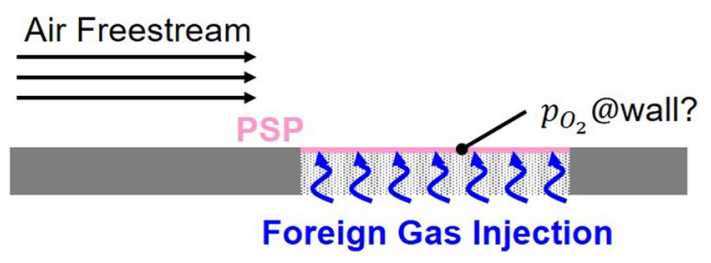

\section{Introduction}

Transpiration cooling is an active thermal protection system with the potential to be useful for aerospace applications in high-speed vehicles, rocket nozzles and future turbine components. A coolant is fed through a porous wall and forms a protective film upon exiting. This coolant film acts as a barrier against the hot freestream, thereby reducing the aerothermal heating (Ifti et al. 2018). In high-enthalpy environments, the film mitigates catalytic heating and surface oxidation by

Marc Ewenz Rocher

marc.ewenzrocher@eng.ox.ac.uk

1 Department of Engineering Science, Oxford Thermofluids Institute, University of Oxford, Oxford, UK

2 Department of Mechanical, Aerospace and Civil Engineering, University of Manchester, Manchester, UK

3 Department of Materials, Faculty of Engineering, Imperial College London, London, UK inhibiting the diffusion of activated species, such as atomic oxygen, through to the surface (Ewenz Rocher et al. 2019b).

When investigating the mass transfer on transpirationcooled surfaces, there are two measurements of interest: the surface pressure, and the coolant concentration. The surface pressure distribution directly influences the pressure differential across the porous medium and, therefore, the coolant mass flux distribution on its surface. The coolant concentration provides a deeper insight into the coolant path after exiting the porous wall and how it mixes with the hot external gas.

Despite their significance, there are currently no methods to measure the surface pressure, nor the coolant distribution, on transpiration-cooled surfaces. Thus, most studies have focused on the downstream effects of injection (Hermann et al. 2018; Han and Rallabandi 2010) or film cooling, whereby the PSP was applied on the solid surface in between the discrete holes (Ahn et al. 2005; Bashir et al. 2017). This 
work investigates a novel pressure-sensitive paint diagnostic to measure the coolant distribution on a transpiration-cooled surface. This method can be used to measure the mass transport from the freestream to the surface and thus to assess the effectiveness of protective coolant films in mitigating oxidation and catalytic heating.

Pressure-sensitive paint (PSP) is sprayed onto the sample without affecting its permeability. The porous alumina substrate bonds the PSP by intermolecular and electrostatic forces. This work builds upon a previous successful application of PSP to anodised aluminium with no cooling feed (Kameda et al. 2004; Gregory et al. 2008) and preliminary tests with transpiration-cooled anodised aluminium, which provided qualitative data (Ewenz Rocher et al. 2019a). Tests were conducted in the Oxford High Density Tunnel (HDT) (McGilvray et al. 2015), using a flat plate model with a porous alumina insert. The results include pressure maps of the sample with air, nitrogen, and no injection, as well as the oxygen partial pressure ratio at a blowing ratio of $F=0.016-0.078 \%$. Laminar and turbulent freestream conditions were explored and compared against each other.

\section{Pressure-sensitive paint on porous alumina}

Pressure-sensitive paint is an optical method to measure the partial pressure of oxygen. It consists of light-emitting molecules dispersed in an oxygen-permeable binder. These molecules are called luminophores and are excited into a higher energy state when illuminated by a light source at a certain wavelength. As they relax into a lower energy state, they will emit light at a different wavelength. In the presence of oxygen, part of this relaxation happens in a non-radiative way, in a process called quenching. More details on the working principle of PSP can be found in Gregory et al. (2008). This change in brightness can be recorded by a camera, with corresponding optical filters. If the gas composition is known, for instance, when injecting air into an air freestream, then the PSP signal scales directly with the local static pressure on the surface. Alternatively, if the pressure field is known, then the $\mathrm{O}_{2}$ partial pressure measured by the PSP can be used to obtain the local concentration of a foreign gas injected into an air freestream.

The specific type of PSP used in this experiment is known as anodised aluminium PSP. Its distinguishing feature is that it does not require a binder. Instead, the luminophores can be sprayed directly onto a surface of porous alumina. This surface is typically created by anodising dense aluminium to create a thin oxidation layer, which has the right pore structure to bond PSP. In the present case, the substrate already consisted of porous alumina, thus the anodisation procedure was not necessary. Instead, $0.1 \mathrm{mmol}$ of
Ruthenium-tris(4,7-diphenyl-1,10-phenanthroline) dichloride, also known as $\left[R u\left(\mathrm{dpp}_{3}\right)\right]^{2+}$, were diluted in $200 \mathrm{ml}$ of dichloromethane and sprayed directly onto the surface after it had been degreased in an ethanol bath. The $\left[R u\left(\mathrm{dpp}_{3}\right)\right]^{2+}$ luminophore was chosen for its stable luminescence and fast response time (Kameda et al. 2004). It furthermore exhibited good signal quality in previous studies (Gregory et al. 2008; Quinn et al. 2011).

The porous alumina substrate was custom-made by Imperial College London. Alumina powder with a particle diameter of $6.45 \mu \mathrm{m}$ was sintered at $1700{ }^{\circ} \mathrm{C}$ for $3 \mathrm{~h}$, yielding a $39 \%$ porous substrate. Its front and back surfaces were polished to open up the pores. The resulting sample combines the ability to bond PSP with the pore size and therefore outflow homogeneity of candidate materials for transpiration cooling, such as $\mathrm{ZrB}_{2}$ (Ifti et al. 2018). As depicted in Fig. 1, the pores of this sample feature a diameter of approximately $2 \mu \mathrm{m}$. More importantly, Fig. 2 shows that the particles do not agglomerate or otherwise block the pores, leaving the permeability unchanged. This was confirmed by permeability measurements before and after spraying the PSP on the sample.

\section{Experimental model}

A flat plate geometry was chosen to validate the PSP diagnostic method as it provides a well-characterised flow field. It is a modified version of the flat plate used in Hermann et al. (2018). Figure 3 presents the main features of the 580$\mathrm{mm}$ long and $125-\mathrm{mm}$ wide plate. 3D printed trips can be inserted into a trip pocket $25.7 \mathrm{~mm}$ downstream of the leading edge to aid the transition. A flush insert was used for the laminar experiments. The porous sample is located $160-\mathrm{mm}$ downstream of the sharp leading edge. Thin-film arrays were mounted upstream and downstream of the sample to determine whether the flow is laminar or turbulent. Two pressure gauges, one upstream, one downstream of the sample, provided static pressure measurements.

The sample geometry consisted of a 2 -mm thick $39.5 \times$ $39.5 \mathrm{~mm}^{2}$ square on top of a $3-\mathrm{mm}$ thick $60-\mathrm{mm}$ diameter disk, as shown in Fig. 4a. It was silicon sealed into a cover plate with QSil-216, as illustrated in Fig. 4. A layer of quickdrying epoxy was applied around the edges beforehand to prevent the silicone from seeping into the pores. Underneath the cover plate is a plenum with a volume of $62 \times 62 \times$ $35-\mathrm{mm}^{3}$.

The coolant properties in the plenum were measured by an Omega 5TC-TT-KI-40-1M fast response thermocouple and a Kulite HEL 375-35BarA pressure transducer. The data acquisition system consisted of a National Instruments PXIe8135 controller with several PXIe-6368 and PXIe-6363 cards operating at a $200 \mathrm{kHz}$ sample rate. The thermocouple 
Fig. 1 Microscopic images of porous alumina surface at different magnification factors
Fig. 2 Microscopic images of porous alumina surface with pressure-sensitive paint. Neither PSP particles nor agglomerates can be seen
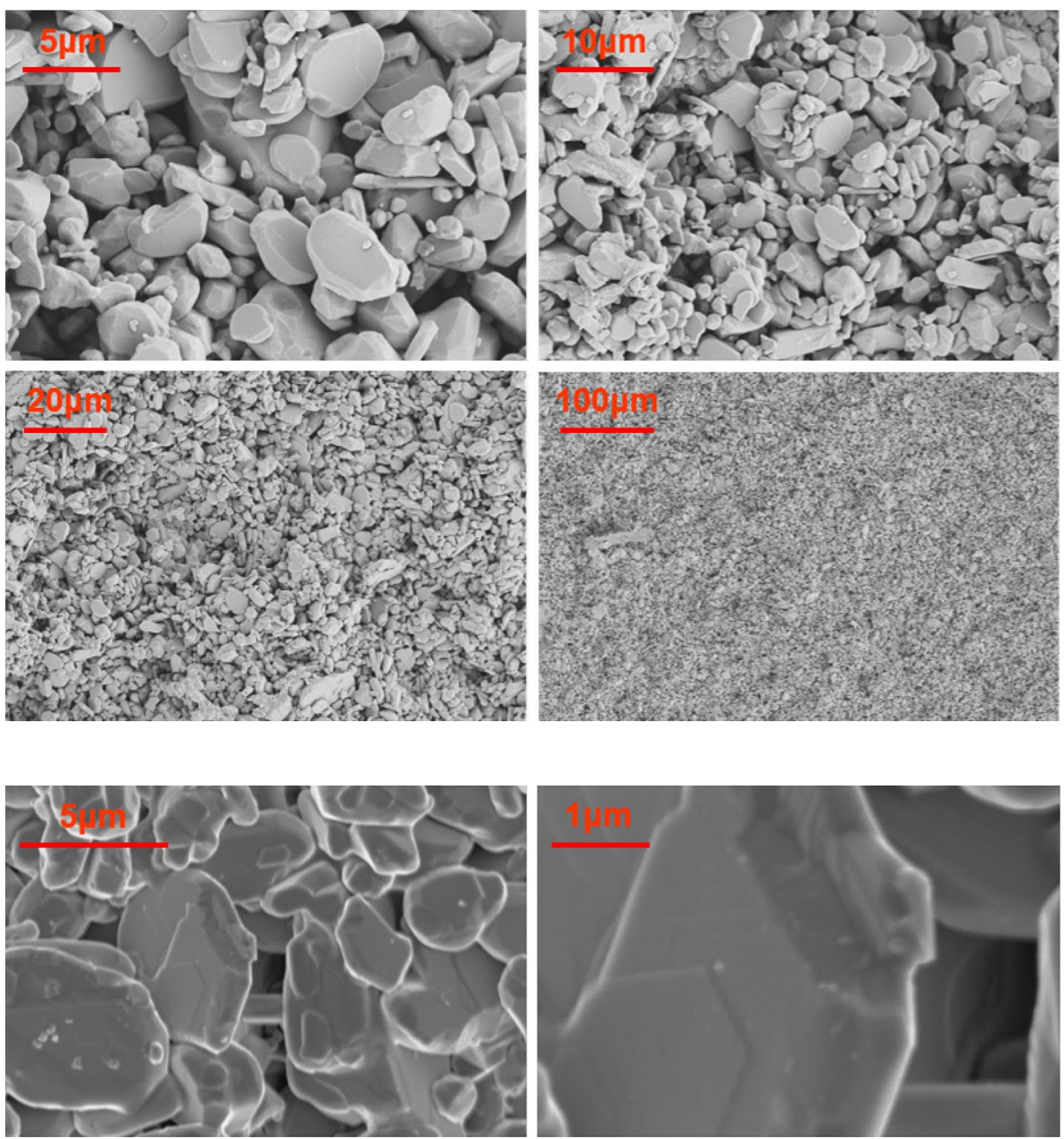

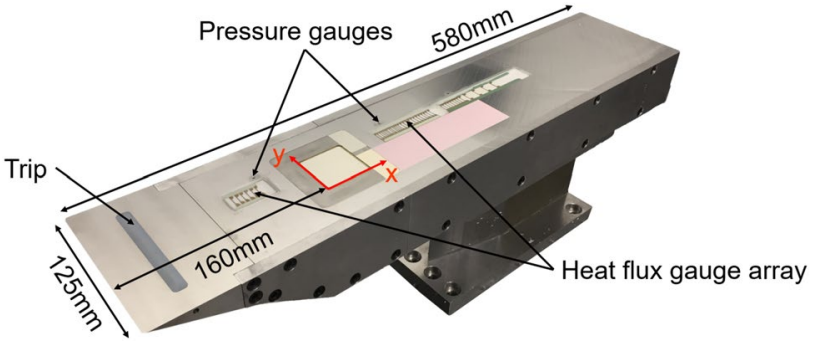

Fig. 3 Photograph of the flat plate model, showing $x$ and $y$ axes in red on the sample

had an uncertainty of $\pm 2.2 \mathrm{~K}$ or $\pm .75 \%$ and the Kulite of \pm $3.5 \mathrm{kPa}$. Two Kulite XCS-093-5A pressure transducers were mounted 55-mm upstream and 65-mm downstream of the sample. They were surface-mounted and positioned on the centreline. The transducers have an accuracy of $\pm 6.3 \%$ of the reading or $\pm 172 \mathrm{~Pa}$, whichever is smaller. A $50 \mathrm{~Hz}$ notch filter coupled with a 300 data points wide moving average filter was employed to remove mains noise.

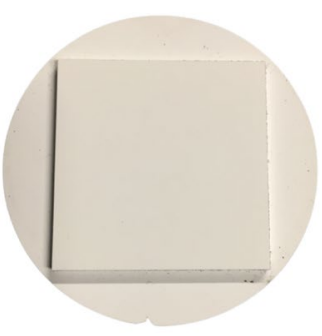

(a) Without PSP

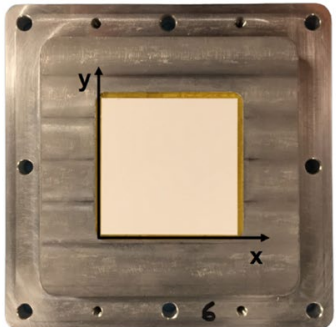

(b) With PSP in cover plate

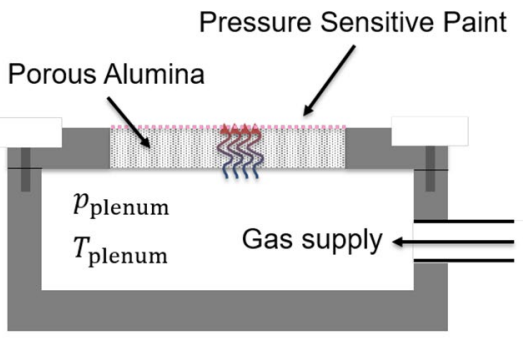

(c) Schematic of plenum

Fig. 4 Illustrations of the porous alumina sample 
Further instrumentation includes platinum thin-film gauges (TFGs) on Macor bodies, which were hand-painted in-house. They were positioned upstream and downstream of the sample and allowed for the measurement of the local heat flux. The electrical resistance of each gauge is temperature-dependent and thus the change in temperature can be measured through the change in voltage if a constant current is applied. A low current of $10 \mathrm{~mA}$ was chosen to prevent Joule heating, and the signal was recorded by an amplifier. The gain profile of the amplifier increases with frequency, which mitigates digitisation errors and reduces the signalto-noise ratio for high-frequency heat flux fluctuations (Anthony et al. 1999). The recorded signals are then postprocessed to remove this frequency-dependent gain, using a method called de-boosting (Oldfield 2008). The gauges were previously calibrated in a water bath at $20-50{ }^{\circ} \mathrm{C}$ in steps of $5{ }^{\circ} \mathrm{C}$, which allows the conversion from voltage to temperature by interpolation. An impulse response convolution method (Oldfield 2008) is subsequently applied to convert the temperature data into heat flux, using the thermal product of Macor: $1705 \mathrm{~J} \mathrm{~m}^{-2} \mathrm{~K}^{-1} \mathrm{~s}^{-0.5}$. This method assumes 1D heat conduction into a semi-infinite block of material. This condition is fulfilled in the short-duration experiments conducted in the Oxford High-Density Tunnel, as the thermal penetration depth is $2.65 \mathrm{~mm}$ into the $7-\mathrm{mm}$ thick material. The resulting heat transfer data is smoothed by a moving average filter with a length of $1 \mathrm{~ms}$.

ISSI fast response PSP was applied next to the thin-film gauges as part of another study that looked at the film effectiveness in the downstream region of a transpiration-cooled surface. It has a characteristic pink hue that is shown in Fig. 3.

A schematic of the optical setup is shown in Fig. 5. A Luminus PT-120-B-L11-EPG LED with a 460-nm dominant wavelength excited the PSP. It was operated at 20 A electric current and featured an in-built thermistor that measured its junction temperature. The absorption and emission spectrum of the $\left[R u\left(\mathrm{dpp}_{3}\right)\right]^{2+}$ luminophores used in this PSP are 400-550 $\mathrm{nm}$ and 600-750 nm, respectively (Gregory et al. 2008). The PSP luminescence is reflected by a mirror before it exits the test section and is captured by a Photron Fastcam

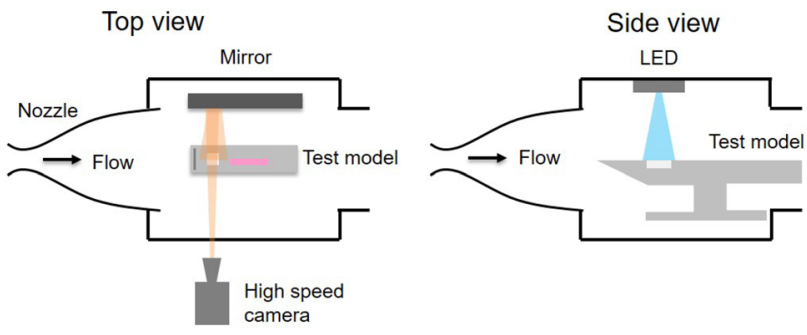

Fig. 5 Schematic of the optical setup
Mini AX 200 900K high speed camera, which performs an auto-shading calibration before every shot. A $550 \mathrm{~nm}$ longpass filter removes any reflected radiation from the LED, ensuring that only the light emitted by the PSP is captured. Figure 6 depicts how the filter effectively blocks the LED light but transmits the PSP signal. The images are recorded at $1000 \mathrm{~Hz}$, with a 1-ms shutter speed, 12-bit depth, and a $1024 \times 1024$ pixel resolution. Using a Tamron SP AF 70-200mm F/2.8 Di LD (IF) macro telephoto zoom lens and a Sigma Apo Tele Converter $2 \mathrm{X}$ Dg for Nikon AF, it was possible to get $758 \times 758$ pixels on the cover plate and $398 \times 398$ pixels on the sample.

The outflow of the sample was characterised pre- and postexperiment. A hot wire was traversed $1.5 \mathrm{~mm}$ above the surface with a coolant pressure drop of 4 bar with no cross-flow using the experimental setup developed in Ifti et al. (2018). The hot wire was calibrated for $0.02-0.5 \mathrm{~m} / \mathrm{s}$ using the StreamLine Pro Automatic Calibrator. The outflow velocity for 4 bar differential pressure is shown in Fig. 7. The velocity field is extremely uniform at $0.045-0.055 \mathrm{~m} / \mathrm{s}$, indicating that the pores are small and evenly distributed. The mass flow rate was measured pre-experimentally with an Alicat M-250SLPM$\mathrm{D} / 5 \mathrm{M}$ mass flow meter with an accuracy of $\pm 0.25 \mathrm{slpm}$. Data

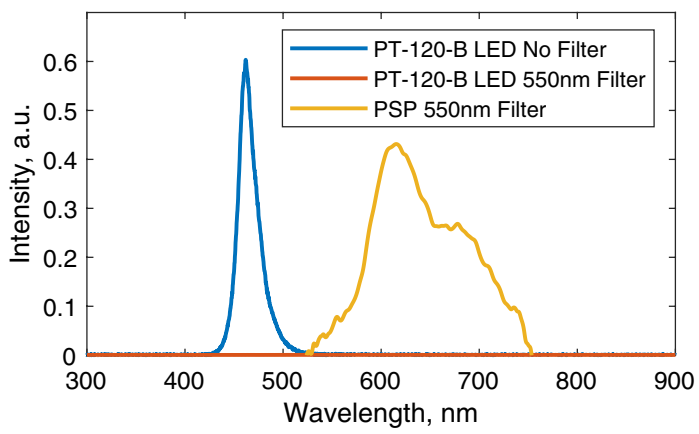

Fig. 6 Comparison of LED and PSP spectra with and without a $550 \mathrm{~nm}$ long-pass filter. Note that the LED intensity with a $550 \mathrm{~nm}$ filter is zero

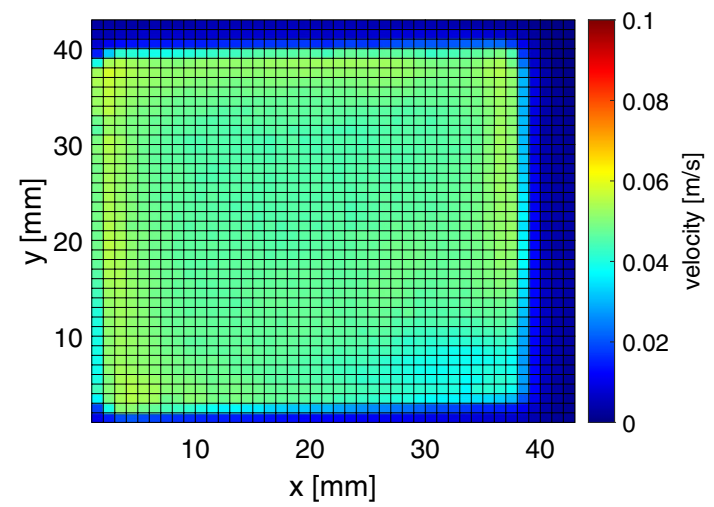

Fig. 7 Outflow characterisation of porous alumina 
points were collected in steps of 0.5 bar for pressure drops between 0-7 bar and normalised by the cross-sectional area to provide the mass flux. The fluid temperature is assumed to be constant across the sample. The resulting graph is interpolated to obtain the average mass flux for a given plenum pressure during testing. The Darcy and Forchheimer coefficients are $K_{\mathrm{D}}=3.9 \times 10^{-15} \mathrm{~m}^{2}$ and $K_{\mathrm{F}}=2.8 \times 10^{-10} \mathrm{~m}$, respectively.

\section{Experimental facility and flow conditions}

The experiments in this paper were conducted in the OxfordHigh Density Tunnel, operated in Ludwieg Tunnel mode. A more extensive description of the facility can be found in McGilvray et al. (2015). Six conditions were investigated at a range of unit Reynolds numbers, from laminar to turbulent. The boundary layer edge conditions can be found in Table 1 .

The blowing ratio, $F$, is the ratio of coolant mass flux to mass flux at the boundary layer edge:

$F=\frac{\rho_{\mathrm{c}} v_{\mathrm{c}}}{\rho_{\mathrm{e}} v_{\mathrm{e}}}$,

with $\rho$ and $v$ being the density and velocity, respectively, while the subscripts $\mathrm{c}$ and e denote the coolant and the boundary layer edge. The coolant mass flux is the ratio of coolant mass flow rate, $\dot{m}$, and through-flow area, $A=39.5 \times 39.5 \mathrm{~mm}^{2}$ :

$\rho_{\mathrm{c}} v_{\mathrm{c}}=\frac{\dot{m}}{A}$.

The relationship between mass flow rate and plenum pressure is characterised pre- and post-experiment as described in Sect. 3. The boundary layer edge mass flux is obtained as follows:

$\rho_{\mathrm{e}} v_{\mathrm{e}}=\frac{p_{\mathrm{e}}}{R T_{\mathrm{e}}} M_{e} \sqrt{\gamma R T_{\mathrm{e}}}$,

where $p_{\mathrm{e}}$ is the static pressure recorded by the surfacemounted Kulite in the no injection case and $\gamma, R$ and $M$ denote the heat capacity ratio, the specific gas constant for air, and the Mach number.

Table 1 Overview of test conditions

\begin{tabular}{lllll}
\hline Description & Mach & $T_{0}(\mathrm{~K})$ & $p_{\mathrm{e}}(\mathrm{kPa})$ & $\operatorname{Re}_{\mathrm{u}}\left(10^{6} / \mathrm{m}\right)$ \\
\hline L1 (laminar) & 6.7 & 435 & 0.95 & 25.8 \\
L2 (laminar) & 6.7 & 437 & 1.02 & 27 \\
L3 (laminar) & 6.7 & 440 & 1.08 & 28 \\
T1 (turbulent) & 5.7 & 342 & 2.14 & 46 \\
T2 (turbulent) & 5.7 & 350 & 2.30 & 48 \\
T3 (turbulent) & 5.7 & 360 & 2.53 & 50 \\
\hline
\end{tabular}

A summary of the outflow conditions is provided in Table 2.

\section{PSP signal post-processing}

The signal emitted by the PSP is reflected by a mirror inside the test section before being captured by the camera, as illustrated in Fig. 5. As the running of the facility induces a vibration on the mirror, the raw image must be stabilised. This is achieved with a single-point stabilisation that corrects for horizontal and vertical translation with respect to a reference frame. A two-point stabilisation can correct for rotation and stretching too, but was found to induce too much noise. The stabilised, raw intensity image is then trimmed to the region of interest: the surface of the transpiration-cooled material. Frames are averaged over the 40-ms long steady test period and normalised by the pre-flow values on a pixel-by-pixel basis.

Note that the intensity value is also dependent on the LED temperature, as illustrated in Figure 8. This effect was corrected using the following empirical relationship:

$I=\frac{I_{\mathrm{raw}}}{1-0.00196\left(T_{\mathrm{LED}}-40^{\circ} \mathrm{C}\right)}$.

Table 2 Blowing parameters and surface heating

\begin{tabular}{lll}
\hline Description & $F(\%)$ & $\Delta T_{\mathrm{PSP}}(\mathrm{K})$ \\
\hline L1 (laminar) & $0,0.053,0.078$ & $0.93,0.19,0.11$ \\
L2 (laminar) & $0,0.051,0.074$ & $0.945,0.203,0.12$ \\
L3 (laminar) & $0,0.049,0.070$ & $0.96,0.22,0.127$ \\
T1 (turbulent) & $0,0.018,0.037,0.060$ & $2.77,2.20,1.76,1.36$ \\
T2 (turbulent) & $0,0.017,0.035,0.057$ & $3.12,2.49,1.99,1.49$ \\
T3 (turbulent) & $0,0.016,0.032,0.053$ & $3.23,2.58,2.06,1.59$ \\
\hline
\end{tabular}

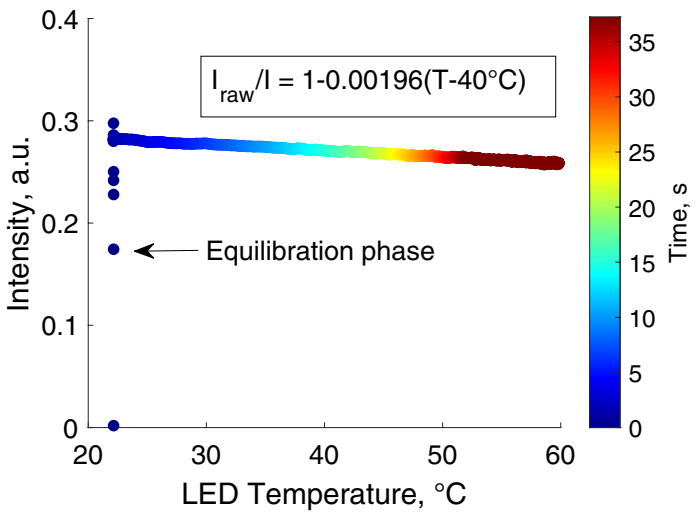

Fig. 8 Intensity versus junction temperature for the PT-120B LED at 20 A electric current 
where $T_{\mathrm{LED}}$ is the LED temperature as recorded by an inbuilt thermistor and raw denotes the raw intensity prior to the LED temperature adjustment. The LED needs about 0.5 $s$ to stabilise thus causing the scattered data points.

The normalised, LED adjusted intensity frames are then converted to pressure using a calibration curve.

\subsection{Calibration}

The raw intensity image can be converted to pressure using a single, global calibration curve that is applied to every pixel, or a pixel-by-pixel calibration whereby each pixel has its own calibration curve. If the image is stable enough, meaning that the position of an individual pixel does not move with time, the pixel by pixel calibration is much more accurate as it accounts for variations in local PSP sensitivity. Due to satisfactory image stabilisation, the pixel-by-pixel calibration was chosen to post-process all normalised intensity images in this work.

The calibration is performed before the experiment, at room temperature without any cross-flow. The intensity of every pixel is recorded while increasing the static pressure in the test section, yielding its individual calibration curve:

$\frac{I_{\mathrm{ref}}}{I}=\left(a \frac{p}{p_{\mathrm{ref}}}+1\right)^{b}$

where $a$ and $b$ are the fitting parameters, while $I$ denotes signal intensity and the subscript ref stands for a reference intensity at a known pressure. The surface temperature remains constant at all times, and all changes in PSP intensity are due to pressure only. Therefore, any PSP intensity signal processed with this calibration curve must be free of temperature effects too. However, the PSP luminophores are heated during the wind tunnel operation. Their intensity will therefore change due to pressure and temperature. This thermal effect can be accounted for if the temperature of the $\mathrm{PSP}, T_{\mathrm{PSP}}$, is known during the test time.

\subsection{PSP temperature}

\subsubsection{No blowing}

When there is no mass injection $(F=0)$, then the pressure across the sample is relatively constant and can be determined using pressure gauges. While the Kulites are not directly on the sample, one is mounted $55-\mathrm{mm}$ upstream and the other 65-mm downstream of it, as shown in Fig. 3. Despite their different locations, they record the same pressure within a $1 \%$ discrepancy. This pressure profile can be converted into an intensity profile using the calibration curve:
$\frac{I\left(p_{\text {ref }}, T_{\text {ref }}\right)}{I\left(p_{\text {gauge }}, T_{\text {ref }}\right)}=\left(a \frac{p_{\text {gauge }}}{p_{\text {ref }}}+1\right)^{b}$

The difference between $I\left(p_{\text {gauge }}, T_{\text {ref }}\right)$ and the actual intensity measured during the experiment, $I\left(p_{\text {gauge }}, T_{\mathrm{PSP}, F=0}\right)$, is the PSP temperature, $T_{\mathrm{PSP}, F=0}$, which deviates from $T_{\text {ref }}$. Therefore

$\frac{I\left(p_{\text {ref }}, T_{\text {ref }}\right)}{I\left(p_{\text {gauge }}, T_{\mathrm{PSP}, F=0}\right)}=\frac{I\left(p_{\text {ref }}, T_{\text {ref }}\right)}{I\left(p_{\text {gauge }}, T_{\text {ref }}\right)} \frac{I\left(p_{\text {gauge }}, T_{\text {ref }}\right)}{I\left(p_{\text {gauge }}, T_{\mathrm{PSP}, F=0}\right)}$.

where the second term on the right hand side denotes the temperature effect on the PSP. The temperature sensitivity of the present PSP was experimentally obtained in a calibration chamber and was found to be $1.17 \% / \mathrm{K}$ for pressures between $1-3 \mathrm{kPa}$. This yields

$\frac{I\left(p_{\text {gauge }}, T_{\text {ref }}\right)}{I\left(p_{\text {gauge }}, T_{\mathrm{PSP}, F=0}\right)}=1+0.0117\left(T_{\mathrm{PSP}, F=0}-T_{\text {ref }}\right)$

which can be inserted into Eq. (7) to solve for $T_{\mathrm{PSP}, F=0}$.

\subsubsection{Blowing}

For the blowing cases, the temperature rises $\Delta T_{\mathrm{PSP}}=T_{\mathrm{PSP}}-T_{\text {ref }}$ were predicted using the porous impulse response analysis for transpiration cooling evaluation (PIRATE) code (Hermann et al. 2020). This tool calculates the reduction in heat flux with blowing, $\frac{\dot{q}}{q_{0}}$, shown in Fig. 9, which was used to obtain the PSP temperature for a given blowing ratio as follows:

$\Delta T_{\mathrm{PSP}}=\Delta T_{\mathrm{PSP}, F=0} \frac{\dot{q}}{\dot{q}_{0}}$

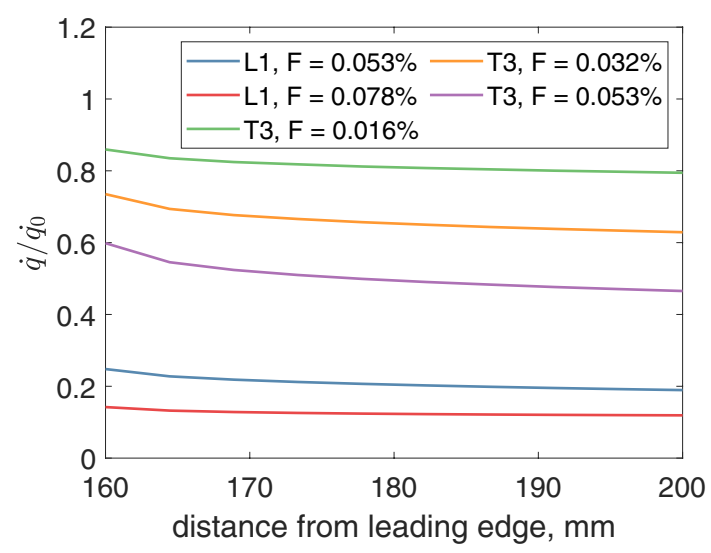

Fig. 9 Heat flux reduction for conditions L1 and T3 at various blowing ratios 
where $\dot{q}$ and $\dot{q}_{0}$ are the heat fluxes with and without blowing, respectively. Combining Eqs. (6)-(8) one obtains for a given temperature rise $\Delta T_{\mathrm{PSP}}$ :

$\frac{I\left(p_{\text {ref }}, T_{\text {ref }}\right)}{I\left(p, T_{\mathrm{PSP}}\right)}=\left(a \frac{p}{p_{\text {ref }}}+1\right)^{b}\left(1+0.0117 \Delta T_{\mathrm{PSP}}\right)$

and for the pressure $p$ :

$p=\left(\left(\frac{I\left(p_{\mathrm{ref}}, T_{\mathrm{ref}}\right)}{I\left(p, T_{\mathrm{PSP}}\right)} \frac{1}{1+0.0117 \Delta T_{\mathrm{PSP}}}\right)^{\frac{1}{b}}-1\right) \frac{p_{\mathrm{ref}}}{a}$.

\section{Results and discussion}

\subsection{Porous interface}

Figure 10 shows the oxygen partial pressure recovered from the PSP signal for condition T3. The signal was spatially averaged over the whole sample. There are 3 blowing rates for air and nitrogen, respectively. The injection always starts before the flow arrival to allow the coolant gas to equilibrate

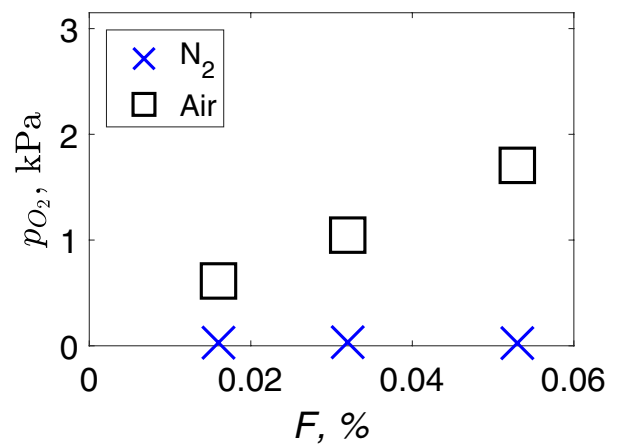

(a) No freestream

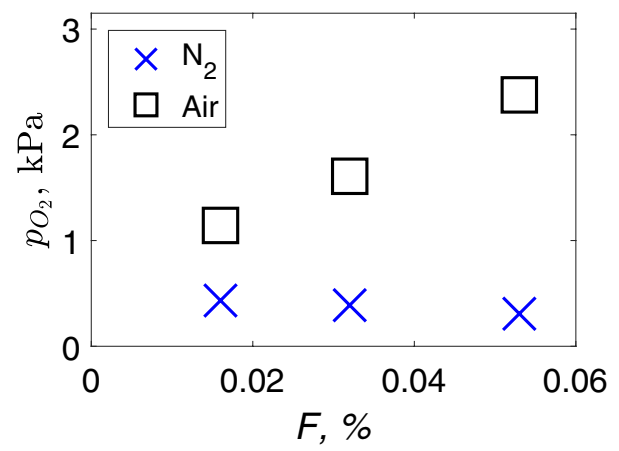

(b) T3 condition

Fig. 10 Oxygen pressure measured by PSP with and without hypersonic cross-flow in the plenum. While there is no noticeable signal change for the nitrogen injection during this time, the oxygen pressure rises drastically for air, reaching the steady-state value shown in Fig. 10a. The fact that there is no change in PSP signal for the nitrogen injection, which has very similar thermal properties to air, rules out temperature effects. Thus, the increase has to be due to oxygen pressure. However, the static pressure in the test section does not change until the flow arrives. The increase in oxygen pressure must be local to the porous interface. As the flow exits the pores, it expands to adjust to the static pressure of the test section. This apparent pressure discontinuity on a macroscopic scale is actually a gradual expansion on a microscopic level, which was also investigated numerically in Rife and di Mare (2019). The PSP molecules that are located in the pores will be surrounded by air with slightly higher pressure than those on the surface. Thus, the air which enters the backside of the sample will not have expanded to the near-vacuum static pressure in the test section yet. This pressure differential is directly related to the depth of the material and can be approximated by the isothermal Darcy-Forchheimer equation. The results are shown in Fig. 11. The effective pore depth calculated with this method is of the same order of magnitude as the $2 \mu \mathrm{m}$ pore diameter observed in Fig. 1. An implication of the porous interface effect is that some PSP particles, deep inside the pores, could become unreachable for the freestream air when the blowing is switched on. The bulk velocity of the injected gas would quickly exceed the diffusion velocity of the freestream air. The magnitude of this effect depends on the distribution of PSP molecules and the microstructure of the porous surface and is difficult to quantify. Qualitatively speaking, the surface will be better protected from freestream oxygen than predicted by theoretical models which assume a flat, homogenoeus surface.

\subsection{Oxygen pressure maps}

Figure 12 shows oxygen pressure maps for the turbulent cases at a range of blowing ratios. The first row of Fig. 12 depicts the no-blowing data $(F=0)$. The signal is spatially uniform, and one can clearly see the contours of the

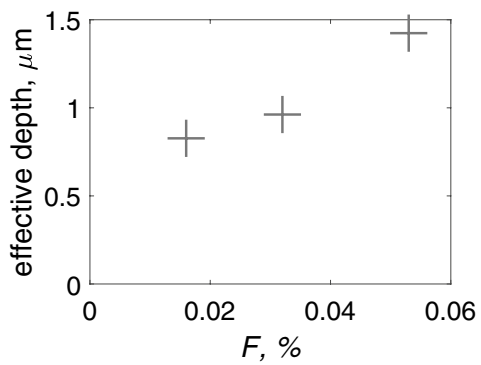

Fig. 11 Effective depth of PSP molecules 
rectangular sample. Note that the bottom right corner was contaminated with epoxy when applying the ISSI porous underlayer to the neighbouring cover plate, causing a small artefact. As expected, the partial $\mathrm{O}_{2}$ pressure increases with the unit Reynolds number due to increasing edge pressure. This trend continues for the blowing cases.

The oxygen pressure decreases with increasing nitrogen injection and decreasing free-stream mass flux. Furthermore, the reduction seems to be slightly larger on the downstream side, which makes sense as the film thickness grows and mitigates oxygen convection and diffusion. It is further noted that the results are much more dependent on the blowing ratio than on the unit Reynolds number.

An uncertainty analysis for the presented data can be found in the Appendix.

\subsection{Oxygen pressure ratio}

Due to different freestream conditions, in particular the static pressure, a direct comparison between laminar and turbulent cases is difficult. It is desirable to obtain a nondimensional parameter, which quantifies how much freestream air and therefore oxygen is displaced by mass injection, as compared to the no injection case. For this purpose, the oxygen pressure with injection is normalised by the oxygen pressure without injection, $\frac{p_{\mathrm{O}_{2} ; \text { inj }}}{p_{\mathrm{O}_{2} \text { :noinj }}}$. Note that injection affects the pressure field. It is therefore theoretically possible that $\frac{p_{\mathrm{O}_{2} \text { inj }}}{p_{\mathrm{O}_{2} \text {;noinj }}}>1$ and hence that injection increases the oxygen concentration at the surface compared to no injection.

Figure 13 shows the oxygen pressure ratio for all blowing ratios of conditions $\mathrm{L} 1$ and $\mathrm{T} 3$. The oxygen pressure ratio decreases with an increasing blowing ratio. Note that the flow was confirmed to be turbulent using the heat flux recorded by the thin film gauges. The bottom row of Fig. 13b and top row of Fig. 13a shows the same blowing ratio in a turbulent $\left(R e_{\mathrm{u}}=5 \times 10^{7}\right)$ and laminar $\left(R e_{\mathrm{u}}=2.58 \times 10^{7}\right)$ flow regime, respectively. The reduction in the oxygen pressure ratio along the sample is much stronger for the laminar case, where mass transport happens through diffusion alone, and the diffusion velocity is directly proportional to the film thickness. As turbulent mixing comes into play at higher unit Reynolds numbers, the coolant film is much less effective at mitigating mass transport from the freestream to the surface. The eddies seem to inhibit the growth of the film, as the oxygen pressure only decreases slightly across the sample.

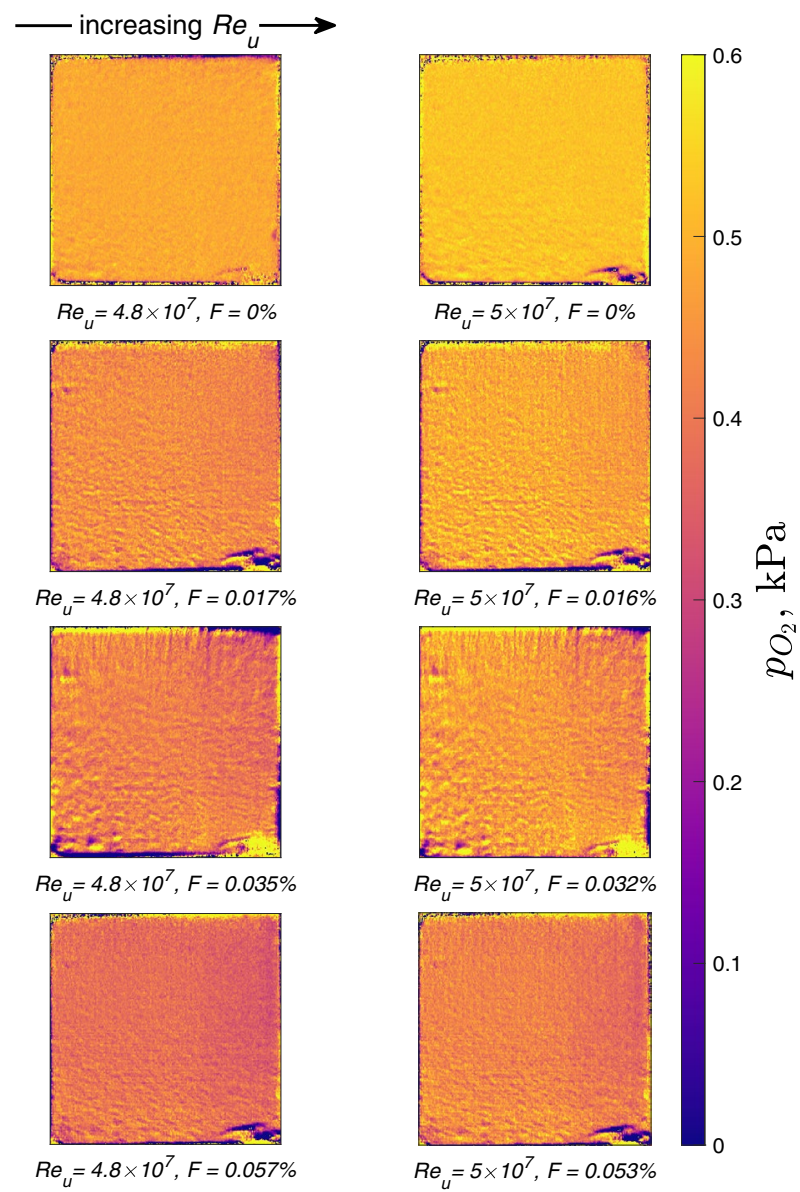

Fig. 12 Oxygen pressure maps for $\mathrm{N}_{2}$ injection at various $R e_{u}$ and $F$ for turbulent conditions. The freestream is flowing left to right.

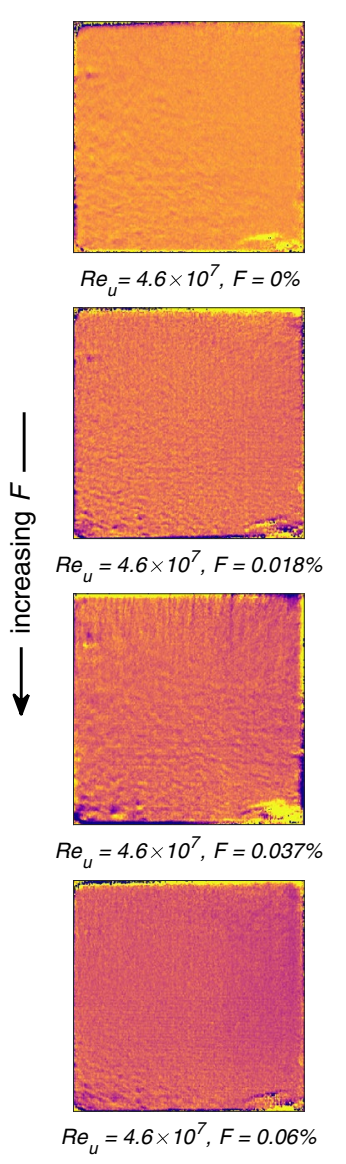




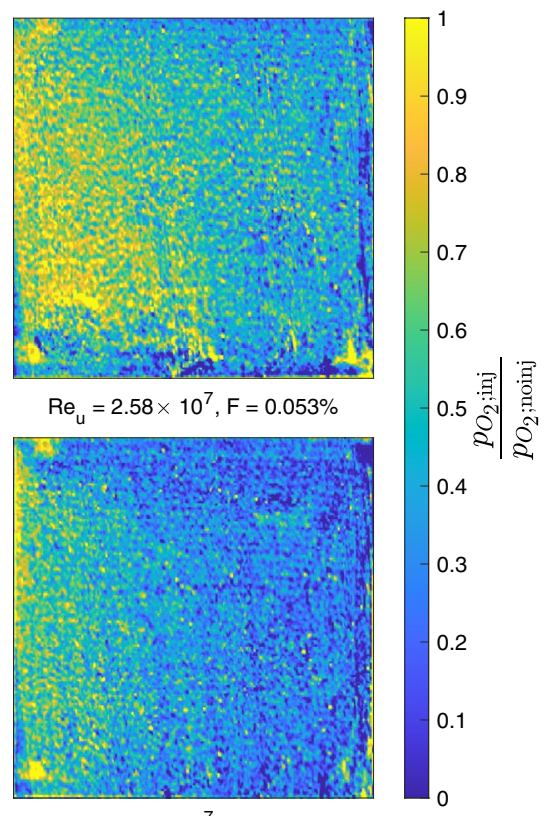

$\mathrm{Re}_{\mathrm{u}}=2.58 \times 10^{7}, \mathrm{~F}=0.078 \%$

(a) laminar condition L1

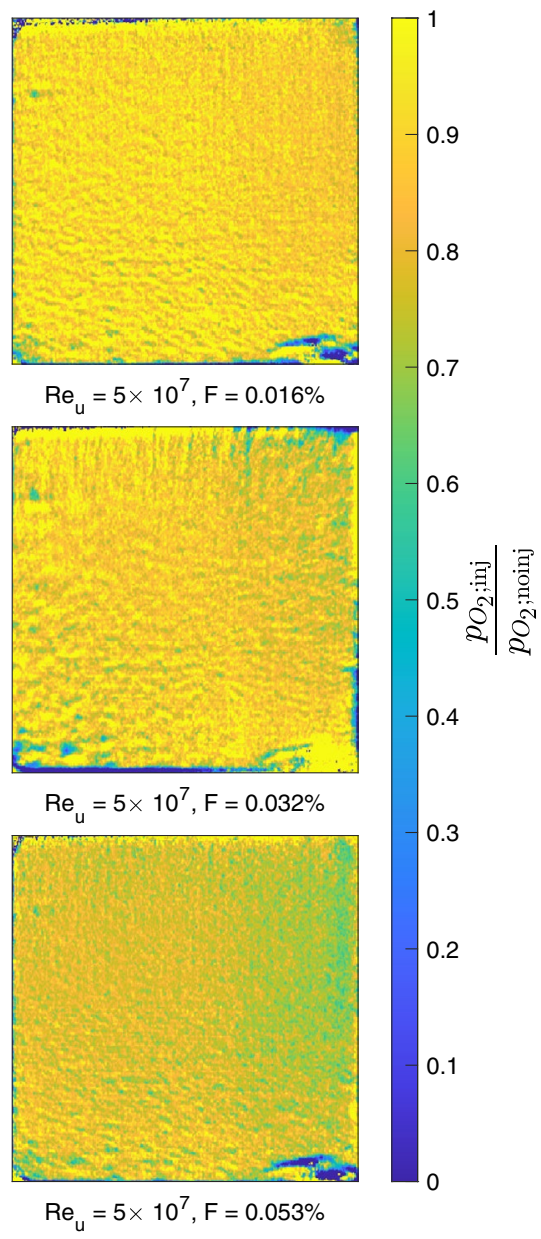

(b) turbulent condition T3

Fig. 13 Spatially resolved oxygen pressure ratio as measured by the PSP

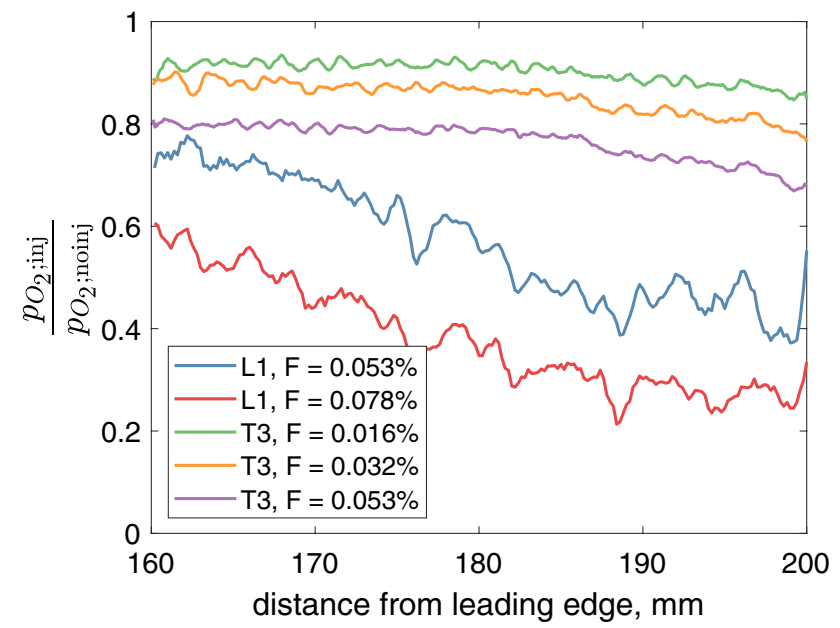

Fig. 14 Comparison of oxygen pressure ratio reduction along the $x$-axis for increasing blowing ratios

\subsection{Spatially averaged oxygen pressure ratio}

Figure 14 depicts the average oxygen pressure ratio for increasing nitrogen blowing ratios. A 100-pixel wide strip in the center was used for this average to avoid 3D effects at the edges. The oxygen pressure ratio for condition T3 at the upstream edge is $92 \%, 88 \%$ and $81 \%$ for blowing ratios of $0.016 \%, 0.032 \%$ and $0.053 \%$, respectively. It falls to $85 \%$, $77 \%$ and $68 \%$ at the downstream edge. The laminar case with $F=0.053 \%$ has a $7 \%$ lower oxygen ratio at the upstream edge than its turbulent counterpart with the same blowing ratio. A much larger discrepancy of $30 \%$ between the two conditions is observed at the downstream edge. The drop in oxygen pressure ratio from upstream to downstream edge for condition L1 is $36 \%$ for both blowing ratios.

The oxygen pressure ratio at the downstream edge for all cases summarised in Table 1, is presented in Fig. 15. An average value along the last $4 \mathrm{~mm}$ in streamwise direction was taken, to reduce noise. The oxygen pressure ratio appears to be linearly dependent on the blowing ratio in the turbulent regime while following a curved trend for the laminar data. The presented experimental data provide a foundation to explore empirical correlations and semi-analytical models for the mass transfer reduction on transpirationcooled surfaces with blowing.

\section{Conclusion}

This work presents a pressure-sensitive paint diagnostic for the species concentration of transpiration-cooled walls. It produced the first spatially resolved oxygen partial pressure map on a porous medium with foreign gas injection. The $\mathrm{O}_{2}$ partial pressure can be converted into $\mathrm{O}_{2}$ 


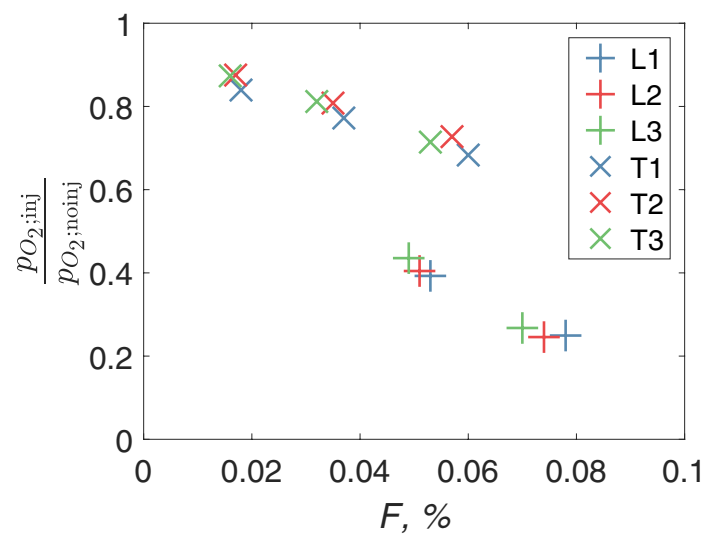

Fig. 15 Average oxygen pressure ratio in the last $4 \mathrm{~mm}$ near the downstream edge plotted against the blowing ratio

concentration at the wall if the static pressure is known. The concentration is critical to investigate how well the porous surface can be protected from oxidation and chemical attack.

By combining the $\mathrm{O}_{2}$ partial pressure maps from the nitrogen injection case, with the $\mathrm{O}_{2}$ pressure recorded in the corresponding no-injection shot, it was possible to compute the oxygen pressure ratio between blowing and non-blowing. Higher blowing ratios and lower Reynolds numbers decrease the oxygen pressure, which is consistent with the literature. The oxygen pressure decreases across the sample, in the direction of the flow, due to the growth of the coolant film. This growth is much more pronounced in the laminar condition, in which mass transfer takes place via diffusion only. Turbulent mixing inhibits the growth of the coolant layer.

The $39 \%$ porous alumina used for this experiment featured a good bond with PSP, while imitating the pore size of Ultra-High-Temperature Ceramics and exhibiting an extremely uniform outflow map. Inexpensive and quick to manufacture, it is a suitable porous substrate to experimentally study transpiration-cooled systems. When the PSP molecules are sprayed onto the porous surface, some fly into pores which are deeper inside the material. If the material is transpiration-cooled, they will experience a higher local pressure than at the surface. Modeling or mitigating this porous interface effect could enable static pressure measurements on transpiration-cooled surfaces in the future.

The following improvements are suggested, to further increase the accuracy of the method. Firstly, filter out temperature effects by coupling every experiment with a nitrogen-freestream case. The obtained thermal response can be deducted from the air-freestream PSP signal. Secondly, improving the image stability by avoiding mirrors in the test section and instead of having a direct optical path between the camera and the sample.

\section{Appendix}

\section{Uncertainty analysis}

The three independent quantities of interest that are measured in this paper are the PSP pressure, the blowing ratio, and the unit Reynolds number. The PSP pressure signal will be affected by PSP degradation and absolute surface temperature. Both effects, however, have been minimised by daily calibrations. The LED intensity correlates with its junction temperature, which is measured by a thermistor. The thermistor is extremely stable, and no drift was observed between calibration runs. Given that all quantities are time-averaged over at least 30 frames, the shot noise for the darkest reading with 600 counts is $\pm 1.49 \%$ per pixel and $\pm 0.15 \%$ for the streamwise spatial averages. The readout noise amounted to $\pm 0.002 \%$ and the dark current was negligible due to frequent auto-shading by the camera. Thus, the PSP temperature, $T_{\mathrm{PSP}}$, remains the main error source.

The unit Reynolds number depends on the static pressure and temperature at the boundary layer edge. The pressure is recorded by a surface-mounted Kulite with an accuracy of $\pm 6.3 \%$ of the reading. This error was found by calibrating the Kulite against an Inficon CDG025D pressure gauge, which itself has an error of $0.2 \%$ of the reading. The static temperature is derived from the total temperature trace, which was established using aspirated thermocouples with an accuracy of $\pm 10 \mathrm{~K}$. The resulting error of the Reynolds number amounts to $\pm 8.7 \%$ for the laminar condition and $\pm 9.3 \%$ for the turbulent condition.

The blowing ratio is calculated using Eqs. (1)-(2), thus again depending on the static temperature and pressure, as well as the mass flow rate. The mass flow meter has a fixed uncertainty of $0.25 \mathrm{slpm}$, thus the error will be smaller for increasing mass flow rates. For the laminar conditions, the resulting error for the blowing ratio amounts to $\pm 24.3 \%$ for the smallest injection pressure, $\pm 18.16 \%$ for medium injection and $\pm 14.8 \%$ for the high injection pressure (Table 2). Equivalently, the blowing ratios in the turbulent condition have an error of $\pm 24.5 \%, \pm 15.5 \%$ and $\pm 14.4 \%$ in ascending order of injection pressure, respectively. By far the largest contributor to this error is the $\pm 6.3 \%$ uncertainty of the surface-mounted pressure gauges. In future experiments, it should be replaced by a more accurate pressure sensor.

Acknowledgements This research is funded by the EPSRC grant "Transpiration Cooling Systems for Jet Engine Turbines and Hypersonic Flight" (reference: EP/P000878/1). The authors of this paper would like to thank Greg King for his dedication and assistance in instrumenting the experimental rig, as well as Maïlys Buquet and Ikhyun Kim for the operation of the High-Density Tunnel. The advice of Imran Naved on the use of PIRATE is gratefully acknowledged. 
Open Access This article is licensed under a Creative Commons Attribution 4.0 International License, which permits use, sharing, adaptation, distribution and reproduction in any medium or format, as long as you give appropriate credit to the original author(s) and the source, provide a link to the Creative Commons licence, and indicate if changes were made. The images or other third party material in this article are included in the article's Creative Commons licence, unless indicated otherwise in a credit line to the material. If material is not included in the article's Creative Commons licence and your intended use is not permitted by statutory regulation or exceeds the permitted use, you will need to obtain permission directly from the copyright holder. To view a copy of this licence, visit http://creativecommons.org/licenses/by/4.0/.

\section{References}

Ahn J, Mhetras S, Han JC (2005) Film-cooling effectiveness on a gas turbine blade tip using pressure-sensitive paint. J Heat Transf 127(5):521-530

Anthony R, Oldfield M, Jones T, LaGraff J (1999) Development of high-density arrays of thin film heat transfer gauges. In: Proceedings of the 5th ASME/JSME thermal engineering joint conference, San Diego, AJTE99-6159

Bashir MH, Shiau CC, Han JC (2017) Film cooling effectiveness for three-row compound angle hole design on flat plate using PSP technique. Int J Heat Mass Transf 115:918-929

Ewenz Rocher M, Hermann T, McGilvray M, Ifti H, Quinn M (2019a) Studying the film effectiveness of transpiration-cooled walls using pressure sensitive paint. In: FAR conference, Monopoli, p 294

Ewenz Rocher M, McGilvray M, Hermann TA, Ifti HS, Hufgard F, Eberhart MF, Meindl A, Loehle S, Giovannini T, Vandeperre LJ (2019b) Testing a transpiration-cooled zirconium-di-boride sample in the plasma tunnel at IRS. In: AIAA Scitech 2019 forum, p 1552
Gregory J, Asai K, Kameda M, Liu T, Sullivan J (2008) A review of pressure-sensitive paint for high-speed and unsteady aerodynamics. Proc Inst Mech Eng Part G J Aerosp Eng 222(2):249-290

Han JC, Rallabandi AP (2010) Turbine blade film cooling using PSP technique. Front Heat Mass Transf 1:013001

Hermann T, Ifti H, McGilvray M, Doherty L, Geraets R (2018) Mixing characteristics in a hypersonic flow around a transpiration-cooled flat plate model. In: 22nd AIAA international space planes and hypersonics systems and technologies conference, $\mathrm{p} 804$

Hermann T, Naved I, McGilvray M (2020) Tool for rapid transient transpiration-cooled reentry simulation. AIAA J 58(2):842-853

Ifti HS, Hermann T, McGilvray M (2018) Flow characterisation of transpiring porous media for hypersonic vehicles. In: 22nd AIAA international space planes and hypersonics systems and technologies conference, p 5167

Kameda M, Tezuka N, Hangai T, Asai K, Nakakita K, Amao Y (2004) Adsorptive pressure-sensitive coatings on porous anodised aluminium. Meas Sci Technol 15(3):489

McGilvray M, Doherty LJ, Neely AJ, Pearce R, Ireland P (2015) The oxford high density tunnel. In: 20th AIAA international space planes and hypersonic systems and technologies conference, $p$ 3548

Oldfield M (2008) Impulse response processing of transient heat transfer gauge signals. J Turbomach 130:021023

Quinn MK, Yang L, Kontis K (2011) Pressure-sensitive paint: effect of substrate. Sensors 11(12):11649-11663

Rife ME, di Mare L (2019) Numerical flux function for flow through porous media with discontinuous properties. J Comput Phys $397: 108833$

Publisher's Note Springer Nature remains neutral with regard to jurisdictional claims in published maps and institutional affiliations. 